\title{
Quantitative estimation of $\alpha$-PVP metabolites in urine by GC-APCI -QTOFMS with nitrogen chemiluminescence detection based on parent drug calibration
}

\author{
Samuel Mesihääa,b,* Ilpo Rasanen ${ }^{\mathrm{b}}$, Ilkka Ojanperä ${ }^{\mathrm{a}, \mathrm{b}}$ \\ a University of Helsinki, Department of Forensic Medicine, P.O. Box 40, FI-00014 University of Helsinki, Finland \\ ${ }^{\mathrm{b}}$ National Institute for Health and Welfare, Forensic Toxicology Unit, Helsinki, Finland
}

\section{A R T I C L E I N F O}

\section{Article history:}

Received 30 October 2017

Received in revised form 14 February 2018

Accepted 17 February 2018

Available online 27 February 2018

\section{Keywords:}

$\alpha$-PVP

Drug metabolite quantification

Gas chromatography

Post-mortem urine

Nitrogen chemiluminescence detection

Time-of-flight mass spectrometry

\begin{abstract}
A B S T R A C T
Gas chromatography (GC) hyphenated with nitrogen chemiluminescence detection (NCD) and quadrupole time-of-flight mass spectrometry (QTOFMS) was applied for the first time to the quantitative analysis of new psychoactive substances (NPS) in urine, based on the N-equimolar response of NCD. A method was developed and validated to estimate the concentrations of three metabolites of the common stimulant NPS $\alpha$-pyrrolidinovalerophenone ( $\alpha$-PVP) in spiked urine samples, simulating an analysis having no authentic reference standards for the metabolites and using the parent drug instead for quantitative calibration. The metabolites studied were OH- $\alpha$-PVP (M1), 2"-oxo- $\alpha-P V P(M 3)$, and N,N-bisdealkyl-PVP (2-amino-1-phenylpentan-1-one; M5). Sample preparation involved liquid-liquid extraction with a mixture of ethyl acetate and butyl chloride at a basic $\mathrm{pH}$ and subsequent silylation of the sechydroxyl and prim-amino groups of M1 and M5, respectively. Simultaneous compound identification was based on the accurate masses of the protonated molecules for each compound by QTOFMS following atmospheric pressure chemical ionization. The accuracy of quantification of the parent-calibrated NCD method was compared with that of the corresponding parent-calibrated QTOFMS method, as well as with a reference QTOFMS method calibrated with the authentic reference standards. The NCD method produced an equally good accuracy to the reference method for $\alpha$-PVP, M3 and M5, while a higher negative bias (25\%) was obtained for $\mathrm{M} 1$, best explainable by recovery and stability issues. The performance of the parent-calibrated QTOFMS method was inferior to the reference method with an especially high negative bias (60\%) for M1. The NCD method enabled better quantitative precision than the QTOFMS methods To evaluate the novel approach in casework, twenty post- mortem urine samples previously found positive for $\alpha$-PVP were analyzed by the parent calibrated NCD method and the reference QTOFMS method. The highest difference in the quantitative results between the two methods was only $33 \%$, and the NCD method's precision as the coefficient of variation was better than $13 \%$. The limit of quantification for the NCD method was approximately $0.25 \mu \mathrm{g} / \mathrm{mL}$ in urine, which generally allowed the analysis of $\alpha$-PVP and the main metabolite M1. However, the sensitivity was not sufficient for the low concentrations of M3 and M5. Consequently, while having potential for instant analysis of NPS and metabolites in moderate concentrations without reference standards, the NCD method should be further developed for improved sensitivity to be more generally applicable.
\end{abstract}

(C) 2018 Elsevier B.V. All rights reserved.

\section{Introduction}

The continuous emergence of new psychoactive substances (NPS) pose a challenge for both toxicological analysis and case interpretation. Among the hundreds of NPS recognized during the ten previous years,

\footnotetext{
* Corresponding author at: University of Helsinki, Department of Forensic Medicine, P.O. Box 40, FI-00014 University of Helsinki, Finland.

E-mail address: samuel.mesihaa@helsinki.fi (S. Mesihää).
}

the stimulant alpha-pyrrolidinovalerophenone ( $\alpha$-PVP) has turned out to be one of the more established drugs on the illicit market despite scheduling as a controlled substance in many countries. $\alpha$-PVP belongs to the "second generation" cathinones that appeared on the illicit market in Europe in 2011 following the "first generation" cathinones 3,4-methylenedioxypyrovalerone (MDPV), 3,4-methylenedioxymethcathinone (methylone), and 4-methylmethcathinone (mephedrone). Current studies suggest that the potency of cathinones to reinforce responding in animal studies, and consequently increased 
abuse liability, is related to their capacity to inhibit dopamine uptake [1]. $\alpha$-PVP has more dopaminergic than serotonergic properties compared with amphetamine analogues [2]. The Risk Assessment carried out by the European Monitoring Centre for Drugs and Drug Addiction reported 116 deaths associated with $\alpha$-PVP between 2012 and 2015 in eight member states [3]. Recently, Swedish authors published a case series of consecutive patients within the STRIDA project with admitted or suspected intake of NPS presenting to hospitals for emergency treatment from January 2011 to March 2016. They detected $45 \alpha$-PVP and 17 MDPV cases in addition to 114 intoxications involving any of 11 other pyrovalerone drugs [4].

Qualitative screening for a broad range of NPS in biological samples is feasible by modern analytical techniques, notably taking advance of the specificity and flexibility of high-resolution mass spectrometry. Accurate mass-based identification of NPS in dataindependent acquisition mode is particularly useful since it enables immediate suspect screening, while subsequent confirmation can be accomplished once a reference standard has been obtained [5]. However, quantification still remains a major challenge. Continuous appearance and disappearance of NPS complicates the acquisition of authentic reference standards because the standards are expensive and difficult to obtain in a sufficiently short period of time. Metabolites of NPS are rarely commercially available, and consequently expensive custom syntheses may be required to include the most important metabolites in the analysis panel.

It is well recognized that the present-day routine analytical method, liquid chromatography electrospray triple quadrupole tandem mass spectrometry (LC-ESI-MS/MS), is at best suitable only for semi-quantitative estimation of metabolite concentrations when using the parent drug for calibration, because of the large variation in responses between the compounds [6]. However, there are non-MS techniques available that provide a more universal response $[7,8]$. Nitrogen chemiluminescence detection (NCD) shows equimolar response to nitrogen that could be exploited in universal quantification of nitrogen containing compounds [9]. In our previous studies $[10,11]$, we introduced a novel platform for simultaneous identification and quantification of NPS in the absence of authentic reference standards. The instrumentation consisted of gas chromatography (GC) coupled to NCD and atmospheric pressure chemical ionization quadrupole time-of-flight mass spectrometry (APCI-QTOFMS). The GC flow was divided in appropriate proportions between NCD and QTOFMS to achieve quantification and identification, respectively. We obtained promising quantitative results by analyzing blood samples that were spiked post-extraction to avoid emphasizing recovery issues [10]. In addition, we were able to show that taking advantage of the accurate mass measurement of the protonated molecule and product ions by GC-APCI-QTOFMS, the obtained MS/ MS spectra were comparable to existing soft-ionization mass spectral libraries [11].

In the present study, our objective was to advance the concept further by developing a quantitative analysis method, based on the GC-NCD-APCI-QTOFMS platform, for $\alpha$-PVP and its three metabolites in urine samples using the parent drug for calibration. Following liquid-liquid extraction, a derivatization step was introduced to avoid undesirable adsorption of polar metabolites in the GC inlet and column [12,13]. Thereafter, post-mortem urine samples previously found positive for $\alpha$-PVP were re-analyzed by the developed method to reveal the metabolite concentrations.

\section{Materials and methods}

\subsection{Chemicals}

$\alpha$-PVP and the N,N-bis-dealkyl-PVP (2-amino-1-phenylpentan1-one; M5) were from Chiron AS (Trondheim, Norway), OH- $\alpha$-PVP (M1) was from Cayman Chemical (Ann Arbor, MI, USA), and 2"-
Oxo- $\alpha$-PVP (M3) from Toronto Research Chemicals (Toronto, ON, Canada). The structures of $\alpha$-PVP and the three metabolites used in the study are shown in Fig. 1. The derivatization agent MSTFA ( $N-$ methyl- $N$-trimethylsilyltrifluoroacetamide) with $1 \%$ TMCS $(2,2,2-$ trifluoro- $N$-methyl- $N$-(trimethylsilyl)-acetamide, chlorotrimethylsilane) was from Thermo Fisher Scientific (Bellefonte, PA, USA).

\subsection{Sample preparation}

Blank urine samples from five healthy volunteers were used for the validation experiments. The blank urines did not contain coeluting nitrogen-containing components that would interfere with quantification at NCD. Blank urine aliquots were spiked to create external calibration curves for MS and NCD quantifications. Each reference standard was dissolved in methanol to obtain a $1 \mathrm{mg} / \mathrm{mL}$ stock solution. The stock solution was diluted to obtain a working solution containing a mixture of $\alpha$-PVP and metabolites M1, M3 and M5 within a concentration range of $0.4-200 \mu \mathrm{g} / \mathrm{mL}$. Blank urine $(1 \mathrm{~mL})$ was spiked with $25 \mu \mathrm{L}$ of the working solution to prepare solutions ranging from 0.01 to $5 \mu \mathrm{g} / \mathrm{mL}$.

Twenty post-mortem urine samples from medico-legal autopsies, previously identified as positive for $\alpha$-PVP, were selected for metabolite quantification. In such samples where the analytes caused a signal saturation to the mass selective detector the urine volume was adjusted to match $0.2-1 \mu \mathrm{g} / \mathrm{mL}$ concentration.

The $\mathrm{pH}$ of the urine samples was adjusted by mixing with $400 \mu \mathrm{L}$ of $1 \mathrm{M}$ Tris buffer (pH 11) and $40 \mu \mathrm{L}$ of $1 \mathrm{M} \mathrm{NaOH}$ in a $6-\mathrm{mL}$ centrifuge tube, resulting in a $\mathrm{pH}$ between $10-12$. The mixture was extracted with $500 \mu \mathrm{L}$ of ethyl acetate + butyl chloride $(75+25)$ in a vortex mixer for $2 \mathrm{~min}$. After centrifugation, an aliquot $(100 \mu \mathrm{L})$ of the organic phase was transferred into a conical autosampler vial. For the derivatization procedure, the organic phase was mixed with $40 \mu \mathrm{L}$ of MSTFA $+1 \%$ TMCS silylation reagent, incubated for $15 \mathrm{~min}$ at $50^{\circ} \mathrm{C}$ and mixed prior to GC injection.

\subsection{Instrumentation}

A 7890B Series GC System equipped with a 7693 Automatic Liquid Sampler and a split/splitless injector was coupled through a two-way splitter with makeup gas (He) to an Agilent 6540 UHD Accurate-Mass QTOF mass analyser and a 255 Nitrogen Chemiluminescence Detector (all Agilent Technologies, Santa Clara, CA, USA).

\subsection{Nitrogen chemiluminescence detection}

Pyrolysis of the analytes at NCD was carried out at $900^{\circ} \mathrm{C}$ using a hydrogen flow rate of $4 \mathrm{~mL} / \mathrm{min}$ and an oxygen flow rate of $9.4 \mathrm{~mL} / \mathrm{min}$. Data from NCD was collected at $50 \mathrm{~Hz}$ over the entire course of the analysis. OpenLab CDS Chemstation GC driver A.02.05.021 was used to control GC-NCD.
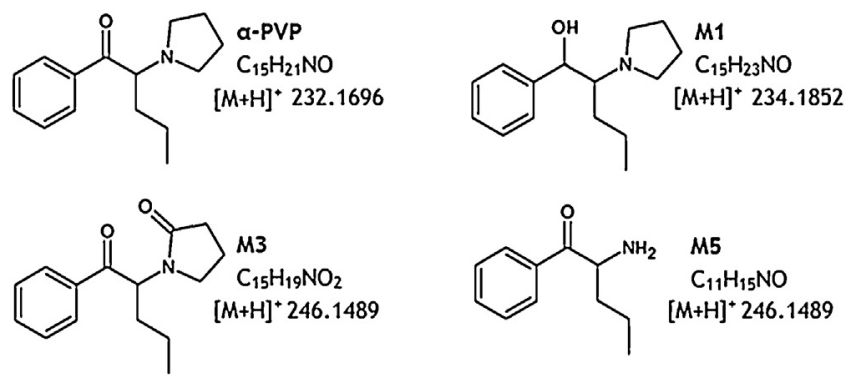

Fig. 1. Chemical structures, molecular formulas and accurate masses of the protonated molecules of $\alpha$-pyrrolidinovalerophenone ( $\alpha$-PVP) and three of its metabolites (M1, M3, M5). 
Table 1

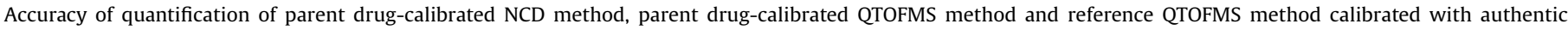
reference standards. ${ }^{a}$

\begin{tabular}{|c|c|c|c|c|}
\hline & Spiked concentration $(\mu \mathrm{g} / \mathrm{mL})$ & Measured concentration $(\mu \mathrm{g} / \mathrm{mL})$ & $\mathrm{CV}(\%)$ & Bias (\%) \\
\hline \multicolumn{5}{|c|}{ GC-NCD calibrated with $\alpha$-PVP } \\
\hline \multirow[t]{2}{*}{$\alpha-P V P$} & 0.25 & 0.26 & 8 & 4 \\
\hline & 1.00 & 0.94 & 4 & 6 \\
\hline \multirow[t]{2}{*}{ M1 } & 0.25 & 0.19 & 13 & 24 \\
\hline & 1.00 & 0.75 & 7 & 25 \\
\hline \multirow[t]{2}{*}{ M3 } & 0.25 & 0.27 & 8 & 8 \\
\hline & 1.00 & 1.09 & 5 & 9 \\
\hline \multirow[t]{2}{*}{ M5 } & 0.25 & 0.29 & 8 & 16 \\
\hline & 1.00 & 1.05 & 5 & 5 \\
\hline \multicolumn{5}{|c|}{ GC-APCI-QTOFMS calibrated with $\alpha$-PVP } \\
\hline \multirow[t]{2}{*}{$\alpha-P V P$} & 0.25 & 0.22 & 18 & 12 \\
\hline & 1.00 & 1.03 & 12 & 3 \\
\hline \multirow[t]{2}{*}{ M1 } & 0.25 & 0.10 & 12 & 60 \\
\hline & 1.00 & 0.42 & 15 & 58 \\
\hline \multirow[t]{2}{*}{ M3 } & 0.25 & 0.24 & 13 & 4 \\
\hline & 1.00 & 1.09 & 14 & 9 \\
\hline \multirow[t]{2}{*}{ M5 } & 0.25 & 0.18 & 12 & 28 \\
\hline & 1.00 & 0.83 & 11 & 17 \\
\hline \multicolumn{5}{|c|}{ GC-APCI-QTOFMS calibrated with authentic reference standards } \\
\hline \multirow[t]{2}{*}{$\alpha-P V P$} & 0.25 & 0.22 & 18 & 12 \\
\hline & 1.00 & 1.03 & 12 & 3 \\
\hline \multirow[t]{2}{*}{ M1 } & 0.25 & 0.25 & 12 & 0 \\
\hline & 1.00 & 1.00 & 15 & 0 \\
\hline \multirow[t]{2}{*}{ M3 } & 0.25 & 0.22 & 13 & 12 \\
\hline & 1.00 & 1.00 & 14 & 0 \\
\hline \multirow[t]{2}{*}{ M5 } & 0.25 & 0.21 & 12 & 16 \\
\hline & 1.00 & 0.94 & 11 & 6 \\
\hline
\end{tabular}

a Averaged values from five different urine samples, each measured in duplicate in six separate days.

External calibration was performed after each data acquisition sequence, using the equimolar nitrogen response to $\alpha$-PVP. Three calibration points $(0.25,1$ and $5 \mu \mathrm{g} / \mathrm{mL})$ were measured in spiked blank urine samples after extraction and derivatization.

The amount of nitrogen was calculated by multiplying the relative amount of nitrogen in the molecule by concentration, dilution factor caused by the derivatization reagent, and injection volume.

\subsection{Gas chromatography}

Injector liner was a Single taper Ultra Inert liner with glass wool (Agilent 5190-2293). Analytical column was a DB-5MS (30 m $\times 0.25 \mathrm{~mm}$ id with $0.1 \mu \mathrm{m}$ film) capillary column (Agilent Technologies). After the analytical column, the GC flow was divided between the NCD and the APCI ion source through a twoway splitter, using $0.55 \mathrm{~m} \times 0.18 \mathrm{~mm}$ and $2 \mathrm{~m} \times 0.18 \mathrm{~mm}$ uncoated deactivated fused-silica post-columns to obtain a 10:1 flow ratio, respectively. The splitter pressure was $15.8 \mathrm{psi}$ and the flow ratio was calculated using the Effluent Splitter Calculator (with Makeup) (Agilent Technologies). In this concurrent detection, the NCD signal is detected 0.02 min earlier than QTOFMS.

GC was operated in the pulsed splitless injection mode with an equilibration time of $0.75 \mathrm{~min}$ and $50 \mathrm{~mL} / \mathrm{min}$ purge flow to split vent at $0.75 \mathrm{~min}$. A pulse pressure of $50 \mathrm{psi}$ for $0.75 \mathrm{~min}$ was applied prior to using initial head pressure of $24.9 \mathrm{psi}$. The injector port temperature was $250{ }^{\circ} \mathrm{C}$ and the transfer line temperature $320^{\circ} \mathrm{C}$. The injection volume was $1.0 \mu \mathrm{L}$. The oven temperature was initially held at $100^{\circ} \mathrm{C}$ for $0.75 \mathrm{~min}$ and then increased by $30^{\circ} \mathrm{C}$ per min to $320^{\circ} \mathrm{C}$, which was held for $6 \mathrm{~min}$. Helium was used as carrier gas at $1 \mathrm{~mL} / \mathrm{min}$ in the constant flow mode.

\subsection{Mass spectrometry}

QTOFMS was operated in APCI positive ionization mode, drying gas (nitrogen) flow at $5.0 \mathrm{~L} / \mathrm{min}$ and gas temperature at $365^{\circ} \mathrm{C}$. Corona discharge needle current was $1000 \mathrm{nA}$ and capillary voltage $1000 \mathrm{~V}$. Fragmentor voltage was $140 \mathrm{~V}$ and skimmer voltage $65 \mathrm{~V}$.

Mass acquisition was performed in All-ions mode, and data were recorded over the $m / z$ range of 50-400 with an acquisition rate of 5 spectra/s. Collision energy in the low energy function was $0 \mathrm{eV}$, whereas in the high energy function 10 and $20 \mathrm{eV}$ were used. External mass calibration was carried out using the APCI tuning mix (Agilent Technologies). Ion $m / z 257.2475$ was used for internal calibration throughout the chromatographic separation. QTOFMS was operated in $2 \mathrm{GHz}$, Extended Dynamic Range mode.

All data were collected with MassHunter Data Acquisition B.04.00 software (Agilent Technologies). MassHunter Profinder B.06.00 software (Agilent Technologies) was used for initial data processing and compound identification.

\subsection{Validation}

Validation was performed according to the recommended guidelines [14], where appropriate. Limit of detection (LOD) was determined using the following criteria for tentative identification: peak area of $>1000$, retention time tolerance of $0.1 \mathrm{~min}$, and mass tolerance of $1 \mathrm{mDa}$. All the quantitative data, including the limit of quantification (LOQ), bias, and precision, were gathered from parallel measurements of five calibration points ranging from 0.01 to $1 \mu \mathrm{g} / \mathrm{mL}$ in spiked control urine samples (Tables 1 and 2). Processed sample stability at room temperature at concentrations of 0.01 and $1.0 \mu \mathrm{g} / \mathrm{mL}$ was tested by measuring the difference from 
Table 2

GC-NCD-APCI-QTOFMS method validation results for $\alpha$-PVP, M1, M3 and M5.

\begin{tabular}{|c|c|c|c|}
\hline Parameter & Detector & Acceptance criteria & Result \\
\hline Bias & NCD & $\pm 30 \%$ & Range from -25 to $16 \%^{\mathrm{a}}$ \\
\hline Carryover & QTOFMS & $<10 \%$ of LOD & No carryover at $10 \mu \mathrm{g} / \mathrm{mL}^{\mathrm{b}}$ \\
\hline LOD & QTOFMS & Identification criteria must be met & $10 \mathrm{ng} / \mathrm{mL}$ \\
\hline LOQ & NCD & Bias and precision criteria must be met & $16 \mathrm{pg} / \mathrm{N} /$ injection $^{\mathrm{c}}, \mathrm{CV}<13 \%$ \\
\hline Precision & NCD & $C V \leq 20 \%$ & Range from 4 to $13 \%$ \\
\hline $\begin{array}{l}\text { Processed sample } \\
\text { stability }\end{array}$ & QTOFMS & $\pm 20 \%$ compared to $t_{o}$ & $>10 \mathrm{~h}^{\mathrm{d}}$ \\
\hline \multirow[t]{2}{*}{ Selectivity } & NCD & No significant peak overlapping & $\begin{array}{l}\text { 9/80 ( } 11 \%) \text { of results by NCD and/or QTOFMS were } \\
\text { disqualified }\end{array}$ \\
\hline & QTOFMS & $\begin{array}{l}\text { Full scan mode: Area of }[\mathrm{M}+\mathrm{H}]^{+} \text {at apex must be }>5 \text {-fold from all other } \\
\text { peaks }\end{array}$ & \\
\hline
\end{tabular}

a Averaged values from five different urine samples, each measured in duplicate in six separate days.

b Averaged values from five different urine samples measured in triplicate.

c Corresponding to approximately $0.25 \mu \mathrm{g} / \mathrm{mL}$ of derivatized M1.

d Averaged values from one urine sample, measured in triplicate in one hour intervals for $10 \mathrm{~h}$.

the averaged time zero $\left(t_{0}\right)$ response within a $10 \mathrm{~h}$ interval. Selectivity was measured from actual post-mortem samples.

\section{Results \& discussion}

\subsection{Method validation with spiked urine samples}

A quantitative analysis method by GC-NCD-APCI-QTOFMS was developed for $\alpha$-PVP and its metabolites M1, M3 and M5 in urine samples. Liquid-liquid extraction was chosen because the technique is rather non-selective and easy to master. Among the several extraction solvents tested, a mixture of ethyl acetate and butyl chloride $(75+25)$ at a basic $\mathrm{pH}$ was found to produce a sufficiently uniform extraction recovery for all the study compounds without excessive background noise.

The sec-hydroxyl- (M1) and prim-amino (M5) metabolites showed initially adsorption and unsymmetrical peaks in GC, and consequently a derivatization step with MSTFA to form trimethylsilyl (TMS) derivatives was included. Compound identification was based on the accurate masses of the protonated molecules for each compound by GC-APCI-QTOFMS. For M1 and M5 the addition of the TMS moiety resulted in a mass shift of $+72.0396 \mathrm{Da}$ to the protonated molecule. Fig. 2 shows a GC-NCD chromatogram from spiked urine samples containing $\alpha$-PVP and metabolites M1, M3 and M5 at two concentration levels.

An experimental setting was designed that simulated a quantitative analysis having no authentic reference standards for the metabolites and using the parent drug instead for quantitative calibration, assuming that the extraction recovery of the parent drug equals that of the metabolites.

Table 1 shows the accuracy of quantification of the parentcalibrated NCD method, compared with that of the corresponding parent-calibrated QTOFMS method and a reference QTOFMS method calibrated with the authentic reference standards. The NCD method produced generally a better quantitative precision than the QTOFMS methods. The NCD method showed an equally good accuracy to the reference method for $\alpha$-PVP, M3 and M5, but a higher negative bias (24-25\%) was obtained for M1. The bias was due to a combined effect of lower extraction recovery and relative instability of the TMS derivative of M1. Cleavage of the TMS derivative was verified as an increase of free M1 with time, and consequently it is recommended to minimize the storage time of the prepared samples prior to analysis. The parent-calibrated QTOFMS method performed worse than the reference method with an especially high negative bias (58$60 \%)$ for M1. This result is obviously due to the differences in the ionization efficiency in the APCI source, in addition to the lower extraction recovery and instability issues.

Acceptance criteria for all method validation parameters determined are summarized in Table 2.

At NCD all compounds are pyrolyzed prior to the chemiluminescent detection. This ensures that the response of most nitrogencontaining compounds is equimolar regardless of the molecular structure or condition, with a notable exception of adjacent nitrogen atoms as pointed out by Yan [9]. The fact that neither of

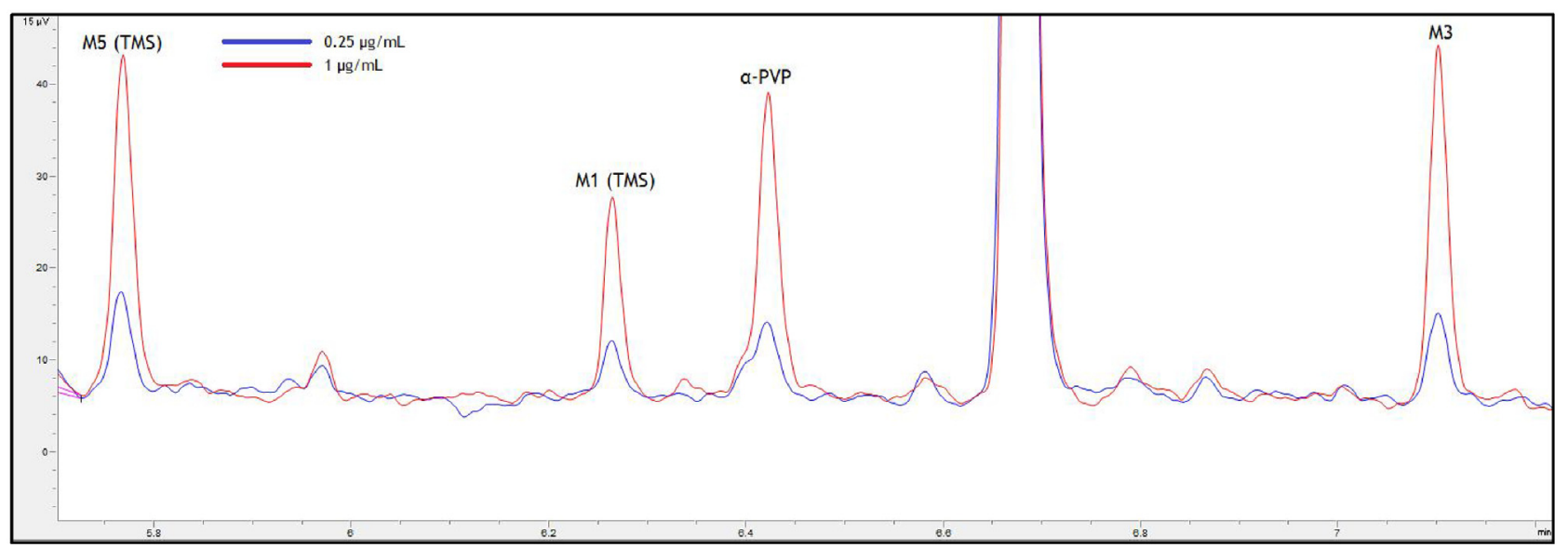

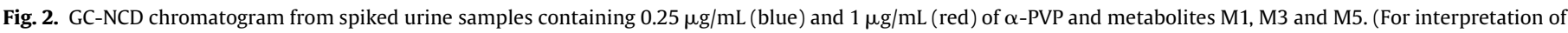
the references to colour in this figure legend, the reader is referred to the web version of this article.) 
Table 3

Comparison of measured drug concentrations in twenty post-mortem urine samples between GC-APCI-QTOFMS calibrated with authentic reference standards and GC-NCD calibrated with $\alpha$-PVP.

\begin{tabular}{|c|c|c|c|c|c|c|c|c|}
\hline \multirow[t]{2}{*}{ Sample } & \multicolumn{2}{|c|}{ a-PVP $(\mu \mathrm{g} / \mathrm{mL})$} & \multicolumn{2}{|c|}{ M1 $(\mu \mathrm{g} / \mathrm{mL})$} & \multicolumn{2}{|c|}{ M3 $(\mu \mathrm{g} / \mathrm{mL})$} & \multicolumn{2}{|c|}{ M5 $(\mu \mathrm{g} / \mathrm{mL})$} \\
\hline & MS & NCD & MS & NCD & MS & NCD & MS & NCD \\
\hline $1^{\mathrm{a}}$ & 8.52 & 8.04 & 6.77 & 5.18 & 0.21 & b & 0.05 & c \\
\hline $2^{a}$ & 4.64 & 5.16 & 4.23 & 3.05 & 0.52 & b & 0.10 & c \\
\hline $3^{a}$ & 3.03 & 3.01 & 0.97 & 0.71 & 0.35 & 0.38 & $<0.01$ & c \\
\hline $4^{\mathrm{a}}$ & 2.93 & 3.01 & 3.66 & 2.92 & 0.63 & 0.70 & 0.06 & c \\
\hline $5^{a}$ & 2.04 & 1.56 & 1.66 & 1.14 & 0.24 & b & 0.04 & c \\
\hline $6^{a}$ & 1.25 & 1.51 & 1.53 & 1.06 & 0.27 & b & 0.03 & c \\
\hline $7^{\mathrm{a}}$ & 1.16 & 1.41 & 0.57 & 0.48 & 0.13 & c & 0.01 & c \\
\hline 8 & 0.99 & 1.20 & 1.47 & 1.00 & 0.05 & c & 0.03 & c \\
\hline 9 & 0.80 & 0.82 & 0.33 & b & 0.08 & c & 0.01 & c \\
\hline 10 & 0.74 & 0.72 & 0.69 & 0.46 & 0.05 & c & $<0.01$ & c \\
\hline 11 & 0.58 & 0.58 & 0.35 & 0.27 & 0.04 & c & $<0.01$ & c \\
\hline 12 & 0.37 & b & 0.27 & 0.18 & 0.19 & b & 0.01 & c \\
\hline 13 & 0.25 & 0.30 & 0.29 & b & 0.03 & c & 0.01 & c \\
\hline 14 & 0.13 & c & 0.05 & c & 0.01 & c & ND & c \\
\hline 15 & 0.10 & c & 0.05 & c & $<0.01$ & c & ND & c \\
\hline 16 & 0.09 & c & 0.14 & c & 0.01 & c & $<0.01$ & c \\
\hline 17 & 0.05 & c & 0.02 & c & $<0.01$ & c & $<0.01$ & c \\
\hline 18 & 0.05 & c & 0.02 & c & $<0.01$ & c & $<0.01$ & c \\
\hline 19 & 0.02 & c & 0.03 & c & ND & c & ND & c \\
\hline 20 & 0.02 & c & 0.57 & b & 0.01 & c & $<0.01$ & c \\
\hline Average bias (\%) ${ }^{\mathrm{d}}$ & & 5.8 & & 26.9 & & 9.9 & & \\
\hline $\mathrm{CV}(\%)^{\mathrm{e}}$ & 12 & 13 & 17 & 8 & 17 & 2 & 18 & \\
\hline
\end{tabular}

a A smaller volume of urine was used to avoid signal saturation at GC-QTOFMS.

b Matrix interference in NCD chromatogram.

c Value below LOQ.

d Average bias (\%) was calculated by comparing MS and NCD measurements.

e CV (\%) was calculated from duplicate measurements. the parent-calibrated methods was capable of fully compensating the extraction recovery of M1 emphasizes the importance of developing a sample preparation method as generic as possible. However, acceptable levels of accuracy were reached using the parent-calibrated NCD method contrary to the parent-calibrated QTOFMS method. Indeed, quantification of metabolites by MS in the absence of authentic reference standards is at best only semiquantitative. A study by Hatsis et al. [6] found up to a 70-fold difference in ion response factor between metabolite and parent drug by ESI-LC-MS/MS.

\subsection{Investigation of post-mortem urine samples}

To evaluate the novel GC-NCD-APCI-QTOFMS platform in casework, twenty post-mortem urine samples previously found positive for $\alpha$-PVP were analyzed by the parent-calibrated NCD method and the reference QTOFMS method. Table 3 shows that by using the parent-calibrated NCD method a quantitative result was obtained in 12 out of $20,11 / 20,2 / 20$ and $0 / 20$ cases for $\alpha$-PVP, M1, M3 and M5, respectively. The highest difference in the quantitative results between the two methods was only 33\%, and the NCD method's precision expressed as the coefficient of variation was better than 13\%. The accuracy and precision of quantification for post-mortem samples was similar to that for the spiked urine samples by the parent-calibrated NCD method (Table 3 ).

M1 and M3 have been considered as the major metabolites of $\alpha$-PVP in humans [15]. In our cases, the presence of these metabolites was confirmed in all but one urine sample by GC-APCIQTOFMS. Currently, the sensitivity of NCD was not sufficient to quantify all metabolites, especially the minor metabolite M5. Moreover, interference caused by co-eluting nitrogen compounds

A

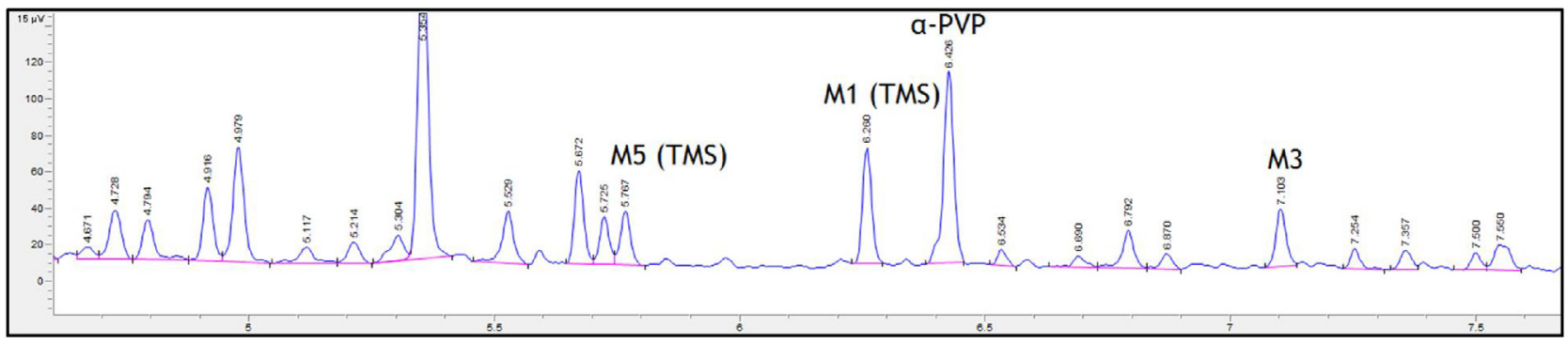

B

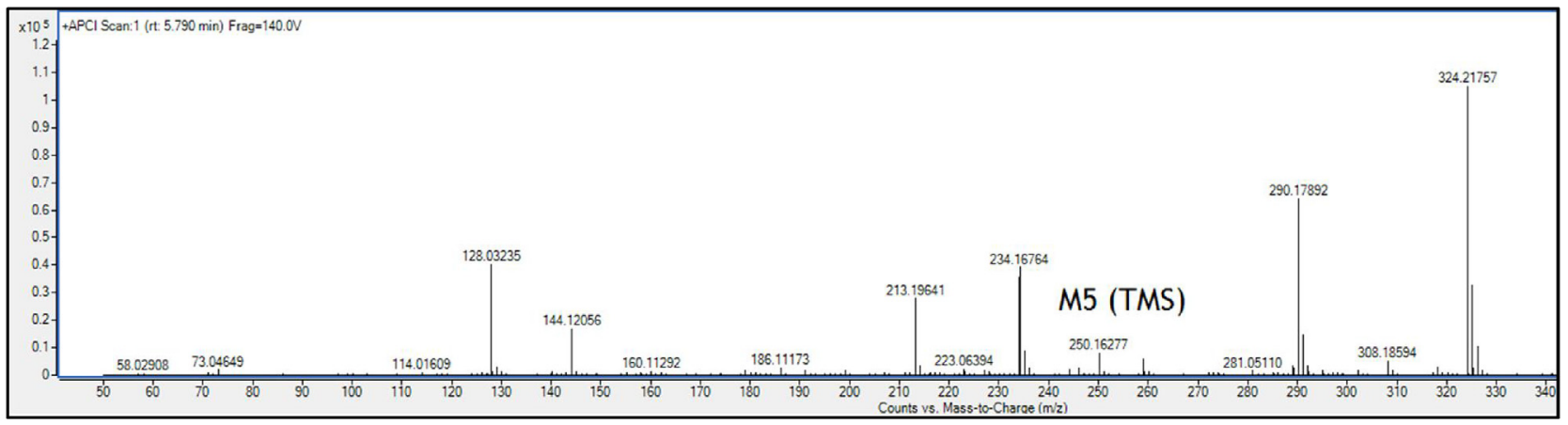

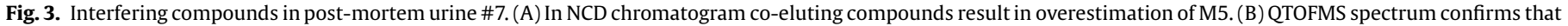
TMS derivatized peak of protonated M5 ( $m / z$ 250.1622) contributes only minimally to total NCD peak area. 
prevented reliable determination in some cases with lower metabolite concentrations (Fig. 3).

According to the manufacturer, the NCD detector can detect nitrogen over the carbon background signal in the mass ratio of $10^{7}$ to one. However, co-eluting nitrogen-containing compounds can cause overestimation in NCD quantification. Such compounds can be due to a multidrug intake or they can be common endogenous urine compounds which are prevalent at low concentrations. In Table 2 we have set criteria for assessing the quality of the quantitative data. For high-sensitivity NCD analysis, common matrix background peaks should be subtracted using a blank urine. A viable strategy to enhance chromatographic selectivity would be using two-dimensional GC [16].

\section{Conclusions}

This study demonstrated that the current GC-NCD-APCI-QTOFMS instrument platform has potential in the rapid quantitative bioanalysis of the main $\alpha$-PVP metabolites together with the parent drug in cases where authentic reference standards are not immediately available. As conventional validated analysis necessitates information on retention time, compound specific spectral fragmentation and quantitative response obtained with an authentic reference standard, these conditions cannot be realized in case of infrequently encountered NPS metabolites. However, identifying NPS metabolites would facilitate the confirmation of the parent drugs, and metabolite quantification can aid forensic interpretation, in particular when investigating the time of drug intake utilizing metabolite to parent drug ratios. In future studies we will seek to improve the NCD method's LOQ using the large volume injection and widen the scope of substances included in the NPS screening.

\section{Compliance with ethical standards}

This article does not contain any studies with living human participants or animals performed by any of the authors. The analysis of drugs from the autopsy specimens was performed according to the request of judicial authorities.

\section{Conflict of interest}

The authors declare that they have no conflict of interest.

\section{Acknowledgement}

The corresponding author (S. M.) thanks the Finnish Cultural Foundation (Suomen Kulttuurirahasto) for a personal research grant.

\section{References}

[1] B.M. Gannon, K.I. Galindo, M.P. Mesmin, A. Sulima, K.C. Rice, G.T. Collins, Relative reinforcing effects of second-generation synthetic cathinones: acquisition of self-administration and fixed ratio dose-response curves in rats, Neuropharmacology (2017), doi:http://dx.doi.org/10.1016/j.neuropharm.2017.08.018 pii: S0028-3908(17)30385-4 [Epub ahead of print].

[2] M. Liechti, Novel psychoactive substances (designer drugs): overview and pharmacology of modulators of monoamine signaling, Swiss Med. Wkly. 145 (January) (2015) w14043.

[3] European Monitoring Centre for Drugs and Drug Addiction, Report on the risk assessment of 1-phenyl-2-(pyrrolidin-1-yl)pentan-1-one ( $\alpha$-pyrrolidinovalerophenone, $\alpha$-PVP) in the framework of the Council Decision on new psychoactive substances, Risk Assessments, Publications Office of the European Union, Luxembourg, 2016.

[4] O. Beck, M. Bäckberg, P. Signell, A. Helander, Intoxications in the STRIDA project involving a panorama of psychostimulant pyrovalerone derivatives, MDPV copycats, Clin. Toxicol. (Phila.) 12 (September) (2017) 1-8.

[5] D. Pasin, A. Cawley, S. Bidny, S. Fu, Current applications of high-resolution mass spectrometry for the analysis of new psychoactive substances: a critical review, Anal. Bioanal. Chem. 20 (June) (2017), doi:http://dx.doi.org/10.1007/ s00216-017-0441-4 [Epub ahead of print] Review.

[6] P. Hatsis, N.J. Waters, U.A. Argikar, Implications for metabolite quantification by mass spectrometry in the absence of authentic standards, Drug Metab. Dispos. 45 (May (5)) (2017) 492-496.

[7] L.E. Magnusson, D.S. Risley, J.A. Koropchak, Aerosol-based detectors for liquid chromatography, J. Chromatogr. A 1421 (July) (2015) 68-81.

[8] J. Viinamäki, I. Ojanperä, Concurrent estimation of metabolite concentrations along with parent drug quantification in post-mortem blood, Forensic Sci. Int. 267 (October) (2016) 110-114.

[9] X. Yan, Unique selective detectors for gas chromatography: nitrogen and sulfur chemiluminescence detectors, J. Sep. Sci. 29 (August (12)) (2006) 1931-1945.

[10] I. Ojanperä, S. Mesihää, I. Rasanen, A. Pelander, R.A. Ketola, Simultaneous identification and quantification of new psychoactive substances in blood by GC-APCI-QTOFMS coupled to nitrogen chemiluminescence detection without authentic reference standards, Anal. Bioanal. Chem. 408 (13) (2016) 3395-3400.

[11] S. Mesihää, R.A. Ketola, A. Pelander, I. Rasanen, I. Ojanperä, Development of a GC-APCI-QTOFMS library for new psychoactive substances and comparison to a commercial ESI library, Anal. Bioanal. Chem. 409 (March (8)) (2017) 2007-2013.

[12] H. Kataoka, Derivatization reactions for the determination of amines by gas chromatography and their applications in environmental analysis, J. Chromatogr. A 733 (May (1-2)) (1996) 19-34.

[13] J. Segura, R. Ventura, C. Jurado, Derivatization procedures for gas chromatographic-mass spectrometric determination of xenobiotics in biological samples, with special attention to drugs of abuse and doping agents, J. Chromatogr. B: Biomed. Appl 713 (August (1)) (1998) 61-90.

[14] Scientific Working Group for Forensic Toxicology (SWGTOX), Scientific Working Group for Forensic Toxicology (SWGTOX) standard paractices for method validation in forensic toxicology, J. Anal. Toxicol. 37 (September (7)) (2013) 452-474.

[15] N. Shima, M. Katagi, H. Kamata, S. Matsuta, K. Sasaki, T. Kamata, et al., Metabolism of the newly encountered designer drug a-pyrrolidinovalerophenone in humans: identification and quantitation of urinary metabolites, Forensic Toxicol. 32 (January (1)) (2014) 59-67.

[16] J. Mommers, E. Ritzen, T. Dutriez, S. van der Wal, A procedure for comprehensive two-dimensional gas chromatography retention time locked dual detection, J. Chromatogr. A 1461 (August) (2016) 153-160. 